\title{
The Variation of the Initial Mass Function in Clusters
}

\author{
K. L. Luhman \\ Harvard-Smithsonian Center for Astrophysics, 60 Garden St., \\ Cambridge, MA 02138
}

\begin{abstract}
I review recent measurements of the initial mass function of stars and brown dwarfs in star-forming regions and open clusters and summarize the implications of these data for theories of star formation.
\end{abstract}

\section{Introduction}

There are processes across a wide range of size scales that could potentially play a role in determining the final mass of a star-like body, including the fragmentation of the clouds of dust and gas out of which stars are born, dynamical interactions of protostars and the competition for material to accrete, and the halting of disk accretion by outflows and winds. Each of these mechanisms should have a distinct influence on the IMF. Therefore, by measuring the IMF, and doing so as a function of star-forming conditions, one can try to identify the processes that regulate the masses of stars and brown dwarfs and are most important in their formation. In the following discussion, I review recent observational results in this area of work.

\section{Previous Work on the IMF}

In the half century since the work of Salpeter, mass functions have been estimated in virtually every known type of stellar population. Unfortunately, even with the large body of work on this subject, reliable constraints on variations of the IMF have proved elusive. In a review of work on the IMF, Scalo (1998) examined IMF slopes from the literature as a function of mass. He found a large scatter in these values with no obvious correlation with the physical properties of the populations, such as galactocentric distance, metallicity, or stellar density. The difficulty of such a comparison is that IMF measurements are prone to systematic errors, poor number statistics, biases and incompleteness - particularly at low masses where the objects are faint and can be preferentially lost from evolved systems - and errors in the conversion from a present day mass function to an IMF. As a result, it is unclear how to interpret these data and use them in testing theories of star formation. To arrive at useful measurements, one must work to achieve accuracy to determine the true shape of the IMF in a given population, precision to reliably compare IMFs from one population to the next and thus check for variations, and completeness to low masses to provide confidence in the validity of any turnover or minimum that appears in a measurement of the IMF. 


\section{Why Measure the IMF at Low Masses?}

Above the mass of the Sun, most measurements of IMFs from the literature can be approximated by power laws that rise from high to low masses. Unfortunately, almost any theory of star formation can produce a power-law form for the IMF, and a slope near Salpeter after arbitrary adjustments to the models. As a result, these data are of limited use in testing model predictions. However, this is not the case at low masses, where the presence of a flattening or turnover in the IMF, the mass at which it occurs, the shape of the IMF to lower masses, and the minimum mass at which star-like bodies can form are all sensitive to the underlying physics of star formation.

\section{Why Measure the IMF in Young Clusters?}

Brown dwarfs are brightest when they are young. As a result, they can be detected down to very low masses and in large numbers in young nearby clusters. The effects of dynamical evolution are minimized in these clusters and both the IMF and the initial conditions of star formation are directly observable in the youngest clusters, unlike any other kind of stellar population one might study. There are also challenges associated with studying the IMF in the youngest clusters, namely the obscuration by dust that hampers optical observations and the uncertainties in deriving the physical properties of very young objects. These issues can be addressed by obtaining data at both optical and IR wavelengths, and by applying all available observational tests and consistency checks when converting the raw data to IMFs.

\section{The IMF in Young Clusters}

\subsection{Taurus and IC 348}

I now describe recent measurements of IMFs in young clusters, starting with the Taurus and IC 348 star-forming regions. Briceño et al. (2002) and Luhman et al. (2003a) surveyed $8.4 \mathrm{deg}^{2}$ in the Taurus star-forming region and arrived at a census of members that was complete for $M / M_{\odot} \geq 0.02$ and $A_{V} \leq 4$. Luhman et al. (2003b) presented a similar census for IC 348 .

Because of the vertical nature of the mass tracks for low-mass stars on the H-R diagram, the spectral types of young objects should be well-correlated with their masses. Very little evolution in temperature is expected between the ages of Taurus and IC 348 ( 1 and 2 Myr), implying virtually identical relations between spectral types and masses for these two populations. In addition, a spectral type is a simple, observable quantity that can be measured to good accuracy with relative ease, particularly at $M$ types. Therefore, I use the distributions of spectral types for IC 348 and Taurus as IMF proxies that can be compared in a straightforward, reliable fashion without the involvement of evolutionary models. The IMF samples for Taurus (Briceño et al. 2002; Luhman et al. 2003a) and IC 348 (Luhman et al. 2003b) are unbiased in mass down to 0.02 and $0.03 M_{\odot}$, respectively, which correspond to types of $\sim \mathrm{M} 9$ and M8 for young ages. The numbers of objects as a function of spectral type in these samples 
are plotted in Figure 1. The distribution for IC 348 reaches a maximum at M5, while primary and secondary peaks appear at K7 and M5 in Taurus. Spectral types of M5 and K7 correspond to masses near 0.15 and $0.8 M_{\odot}$ for ages of a few million years by the models of Baraffe et al. (1998). The spectral type distributions for Taurus and IC 348 provide clear, unambiguous evidence for a significant variation of the IMF with star-forming conditions. Previous studies have noted that a difference of this kind might be present between Taurus and clusters like Orion (e.g., Hillenbrand 1997). However, most previous samples of members of star-forming regions have been derived by combining disparate surveys that utilized biased selection techniques (e.g., $\mathrm{H} \alpha$, IR excess). It was unclear whether the predominance of K7 and M0 stars in Taurus was an accurate reflection of the region or simply a result of incompleteness at later types. The new magnitude-limited membership surveys in Taurus and IC 348 have enabled the first comparison of spectral type distributions in which the samples are complete down to late spectral types and contain relatively large numbers of members.

When the data for the samples in IC 348 and Taurus are transformed to individual masses with evolutionary models, the IMFs in Figure 2 are produced. Because the same techniques and models were employed in converting from data to masses for each population, one can be confident in the validity of any differences in these IMFs. The IMF for Taurus peaks near $0.8 M_{\odot}$ and steadily declines to lower masses. Meanwhile, the mass function for IC 348 rises from high masses down to a solar mass in a roughly Salpeter fashion, rises more slowly down to a maximum at 0.1-0.2 $M_{\odot}$, and then declines into the substellar regime. This spectroscopically determined IMF data agrees well the IMF derived by Muench et al. (2003b) through near-IR luminosity function modeling. Luhman et al. (2003b) quantified the significance of the differences in the distributions of spectral types and masses for Taurus and IC 348 by performing a two-sided Kolmogorov-Smirnov test between the distributions for spectral types of $\leq \mathrm{M} 8$ and for masses of $M / M_{\odot} \geq 0.03$. In terms of both spectral types and masses, the probability that the samples for Taurus and IC 348 are drawn from the same distribution is $\sim 0.01 \%$.

The shapes of the IMFs derived for Taurus and IC 348 are sensitive to the adopted temperature scale and evolutionary models. The combination used here was designed to produce the best agreement with the various observational constraints (Luhman et al. 2003b). In particular, the IMFs above $\sim 0.5 M_{\odot}$ should be fairly accurate since the choices of temperature scale and models implies masses from the H-R diagram that are consistent with the dynamical mass estimates of young stars. However, because fewer constraints are available at low masses, the IMFs below $\sim 0.5 M_{\odot}$ could be subject to systematic errors.

\subsection{Orion}

Even with the extensive spectroscopic work that has been done in Orion, one can define a well-populated sample of members that is complete down to only mid-M types in that region. To extend the IMF in Orion to lower masses, studies have relied on luminosity function modeling (Hillenbrand \& Carpenter 2000; Luhman et al. 2000; Muench et al. 2002). As a result, a comparison of the IMFs in Taurus and IC 348 to data in Orion is less definitive than the comparison between the 


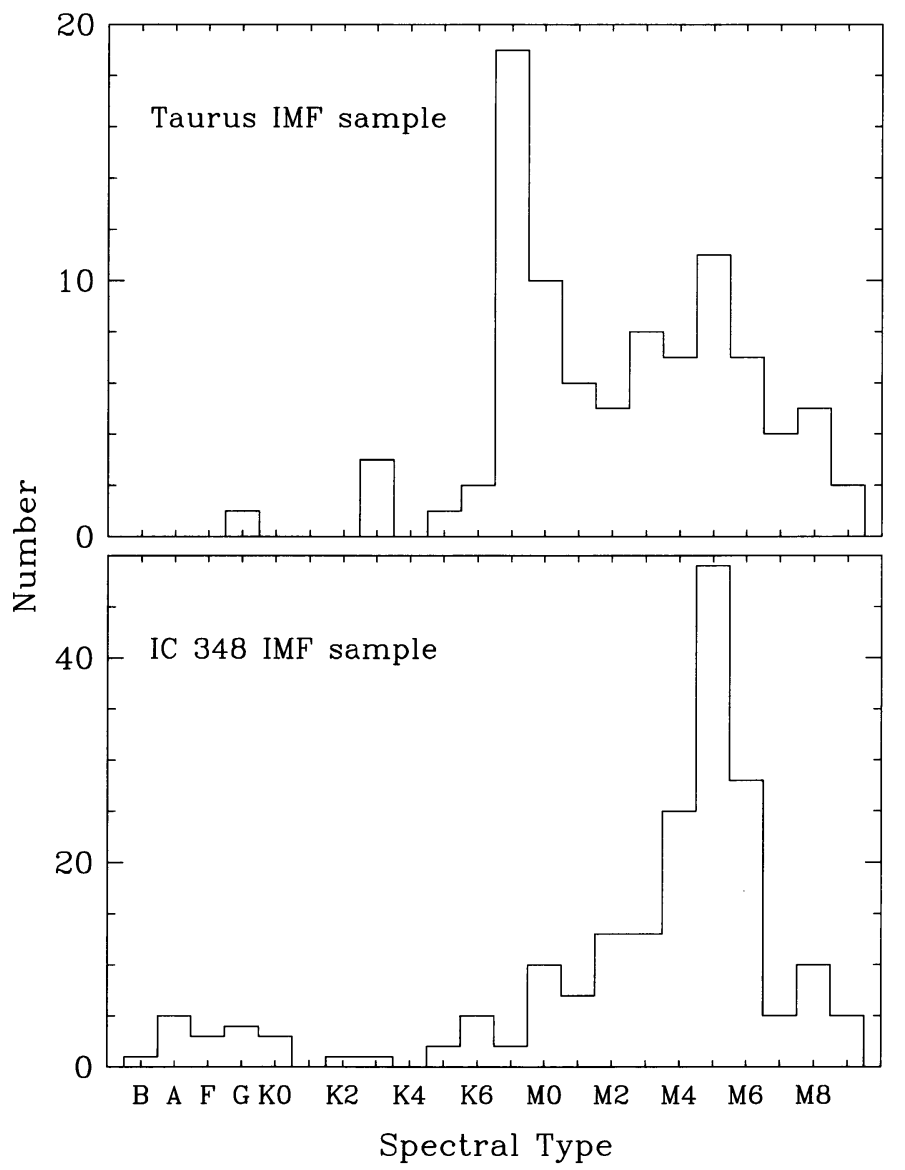

Figure 1. Distributions of spectral types for objects in the IMFs for Taurus (Briceño et al. 2002; Luhman et al. 2003a) and IC 348 (Luhman et al. 2003b) that are shown in Figure 2. These samples are extinctionlimited $\left(A_{V} \leq 4\right)$ and apply to $8.4 \mathrm{deg}^{2}$ in Taurus and to a $16^{\prime} \times 14^{\prime}$ field in IC 348 and are nearly $100 \%$ complete for spectral types of $\leq$ M9 and $\leq \mathrm{M} 8$, respectively. Because the evolutionary tracks for young low-mass stars are mostly vertical, spectral types should be closely correlated with stellar masses. As a result, these distributions of spectral types should directly reflect the IMFs in IC 348 and Taurus. This comparison provides clear, model-independent evidence for significant differences in the IMFs of these two regions. 


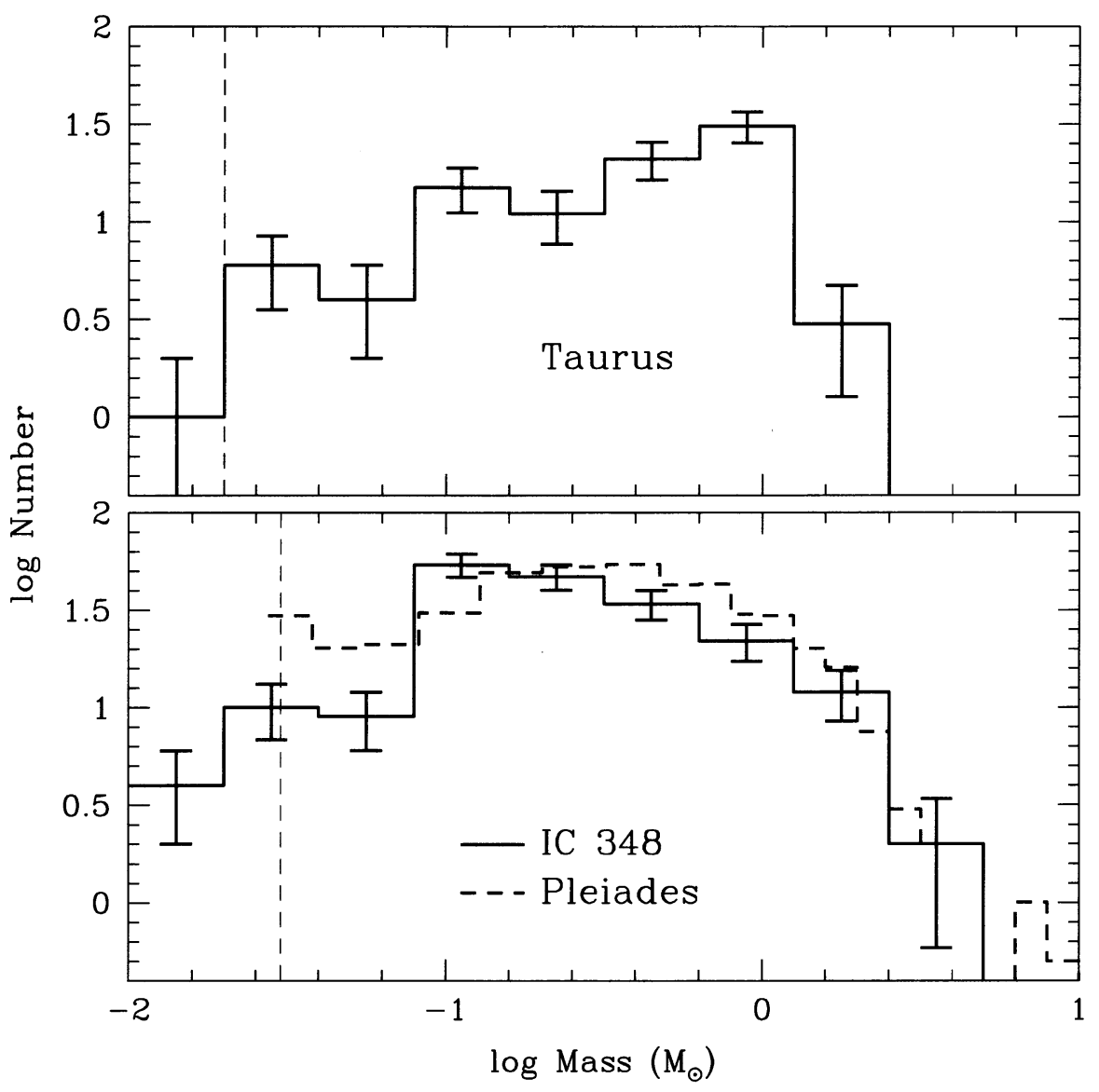

Figure 2. IMFs for extinction-limited samples $\left(A_{V} \leq 4\right)$ in $8.4 \mathrm{deg}^{2}$ in Taurus (Briceño et al. 2002; Luhman et al. 2003a) and in a $16^{\prime} \times 14^{\prime}$ field in IC 348 (Luhman et al. 2003b). These samples are unbiased in mass for $M / M_{\odot} \geq 0.02$ and 0.03 , respectively. The dashed histogram represents the IMF measured for the Pleiades by Moraux et al. (2003). In the units of this diagram, the Salpeter slope is 1.35 . 
first two regions. I consider the IMF reported by Luhman et al. (2000) for the Trapezium Cluster, which was derived with similar methods to the ones used for Taurus and IC 348. The IMF from Luhman et al. (2000) peaks near $0.6 M_{\odot}$ and is roughly flat to lower masses, and thus differs somewhat from the IMF for IC 348 in Figure 2. It is unclear whether this difference is real or is the result of a shortcoming in the merging of the spectroscopic data and the luminosity function modeling for the Trapezium. Meanwhile, the IMFs for IC 348 and the Trapezium derived from luminosity function modeling are similar Muench et al. (2003), but do allow for subtle variations of this type. To reliably compare these IMFs at this level of detail, the completeness limit of the spectroscopic work in the Trapezium must be extended to later spectral types.

\subsection{Pleiades}

In Figure 2, I compare the IMFs for Taurus and IC 348 to a recent measurement for the Pleiades open cluster (Bouvier et al. 1998; Moraux et al. 2003), which reaches a maximum near $0.6 M_{\odot}$, and thus differs from the IMF for IC 348 . Although the IMFs for these two clusters were derived with the same evolutionary models, those models probably have different systematic errors at the two ages in question ( 1 and $100 \mathrm{Myr}$ ), so it is unclear whether this difference between the Pleiades and IC 348 is real. In the next section, I compare these mass functions in terms of the relative numbers of objects in broad mass ranges, which hopefully are less susceptible to systematic errors.

\subsection{Comparison of Relative Numbers in Different Mass Ranges}

In Figure 3, I plot ratios that quantify the relative numbers of high-mass and low-mass stars and the relative numbers of brown dwarfs and stars for IC 348 (Luhman et al. 2003b), Taurus (Briceño et al. 2002; Luhman et al. 2003a), and the Trapezium (Luhman et al. 2000) and for the open clusters of the Pleiades (Bouvier et al. 1998) and M35 (Barrado y Navascués et al. 2001). The latter ratio is not available for the Pleiades and M35 because the surveys in these clusters are not sufficiently complete for brown dwarfs. Four of these populations exhibit comparable relative numbers of high- and low-mass stars, while Taurus produces fewer high-mass stars. Although the IMFs of Taurus and IC 348 are clearly different, they do have comparable numbers of brown dwarfs. Meanwhile, the frequency of brown dwarfs in the Trapezium is double that in the other two regions. Taurus is often referred to as a region of low-mass star formation, but according to Figure 3, it actually favors intermediate mass stars relative to Orion.

\section{Implications for Theories of Star Formation}

The implications of the variations in the IMF among Taurus, IC 348, the Trapezium, and the open clusters for theories of star formation were discussed by Briceño et al. (2002) and Luhman et al. (2003b). They suggested that the lower frequency of brown dwarfs in Taurus and IC 348 relative to the Trapezium and the lower frequency of low-mass stars in Taurus relative to IC 348 could reflect differences in the typical Jeans masses, namely higher values from the Trapezium to IC 348 to Taurus. The model of turbulent fragmentation by Padoan \& 


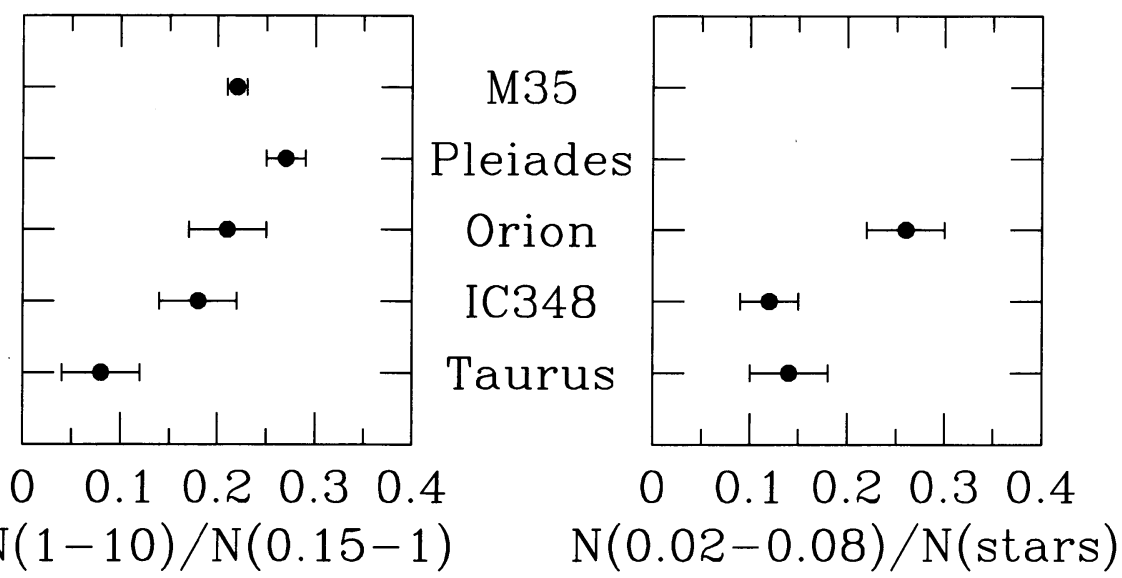

Figure 3. Ratios of the numbers of high-mass and low-mass stars (left) and brown dwarfs and stars (right) for the Taurus, IC 348, and Orion Trapezium star-forming regions and the Pleiades and M35 open clusters.

Nordlund (2002) is an example of a more detailed theory of the IMF that incorporates the Jeans criterion. In their calculations, the IMF peaks at a higher mass for lower values of the gas density and Mach number. Indeed, Taurus exhibits a higher peak mass than IC 348 and is less dense and more quiescent than most star-forming regions. Meanwhile, the observed variations in the IMF would seem difficult to explain with models in which the shape of the IMF is determined by the competition between accretion and outflows (Adams \& Fatuzzo 1996). The significant variation the IMF between Taurus and IC 348 comprises a new and important test for any model of star formation.

\section{References}

Adams, F. C., \& Fatuzzo, M. 1996, ApJ, 464, 256

Baraffe, I., Chabrier, G., Allard, F., \& Hauschildt, P. H. 1998, A\&A, 337, 403

Barrado y Navascués, D., Stauffer, J. R., Briceño, C., Patten, B., Hambly, N. \& Adams, J. 2001, ApJS, 134, 103

Bouvier, J., Stauffer, J. R., Martín, E. L., Barrado y Navascués, D., Wallace, B., \& Bejar, V. J. S. 1998, A\&A, 336, 490

Briceño, C., Luhman, K. L., Hartmann, L., Stauffer, J. R., \& Kirkpatrick, J. D. 2002, ApJ, 580, 317

Hillenbrand, L. A. 1997, AJ, 113, 1733

Hillenbrand, L. A., \& Carpenter, J. M. 2000, ApJ, 540, 236

Luhman, K. L., et al. 2000, ApJ, 540, 1016

Luhman, K. L., Briceño, C., Stauffer, J. R., Hartmann, L., Barrado y Navascués, D., \& Nelson, C. 2003a, ApJ, 590, 348 
Luhman, K. L., Stauffer, J. R., Muench, A. A., Rieke, G. H., Lada, E. A., Bouvier, J., \& Lada, C. J. 2003b, ApJ, 593, 1093

Moraux, E., Bouvier, J., Stauffer, J. R., \& Cuillandre, J.-C. 2003, A\&A, 400, 891

Muench, A. A., Lada, E. A., Lada, C. J., \& Alves, J. 2002, ApJ, 573, 366

Muench, A. A., et al. 2003, AJ, 125, 2029

Padoan, P., \& Nordlund, A. 2002, ApJ, 576, 870

Scalo, J. 1998, in ASP Conf. Ser. 142, The Stellar Initial Mass Function Proceedings, ed. G. Gilmore \& D. Howell, 201 


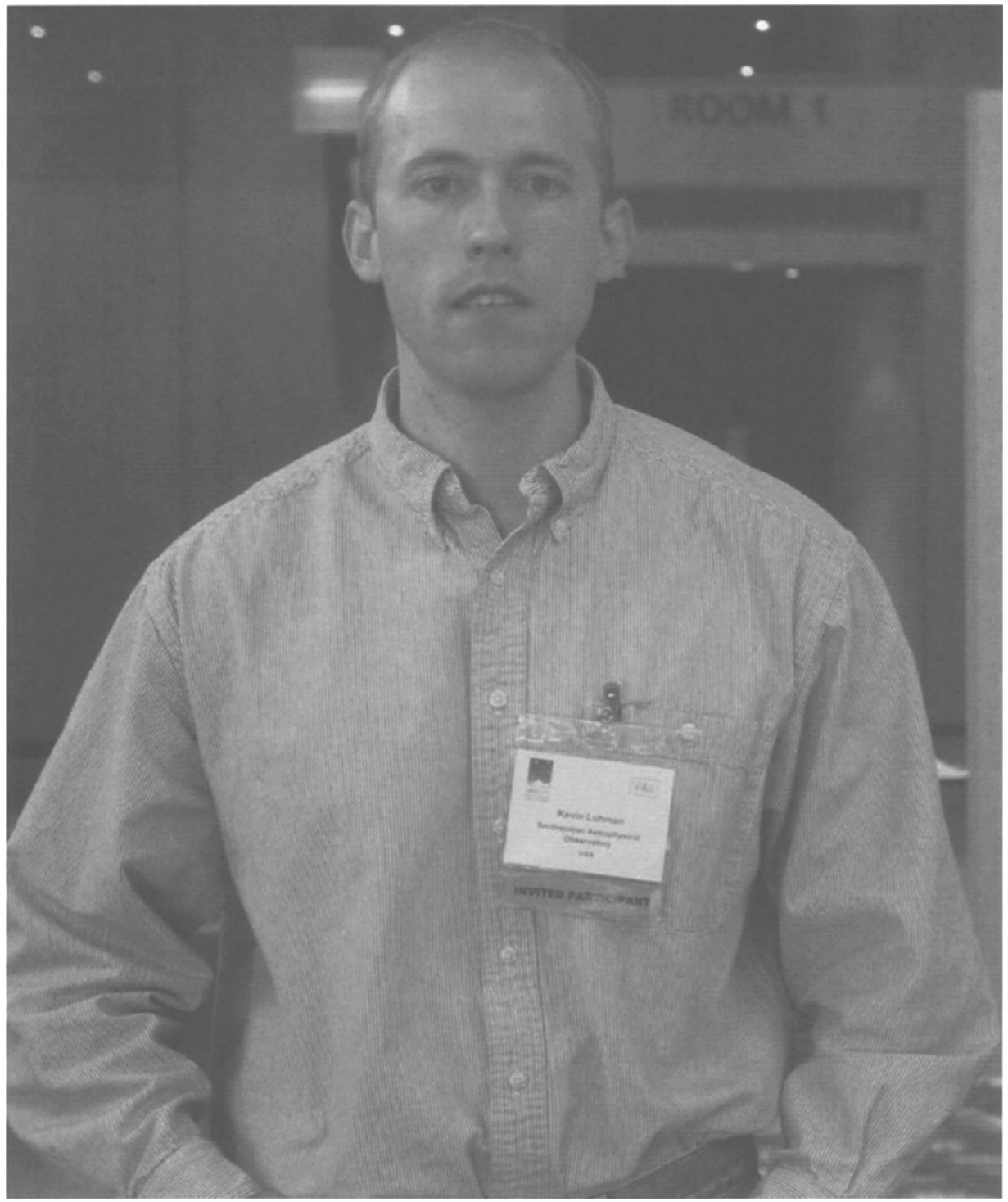

Kevin Luhman 


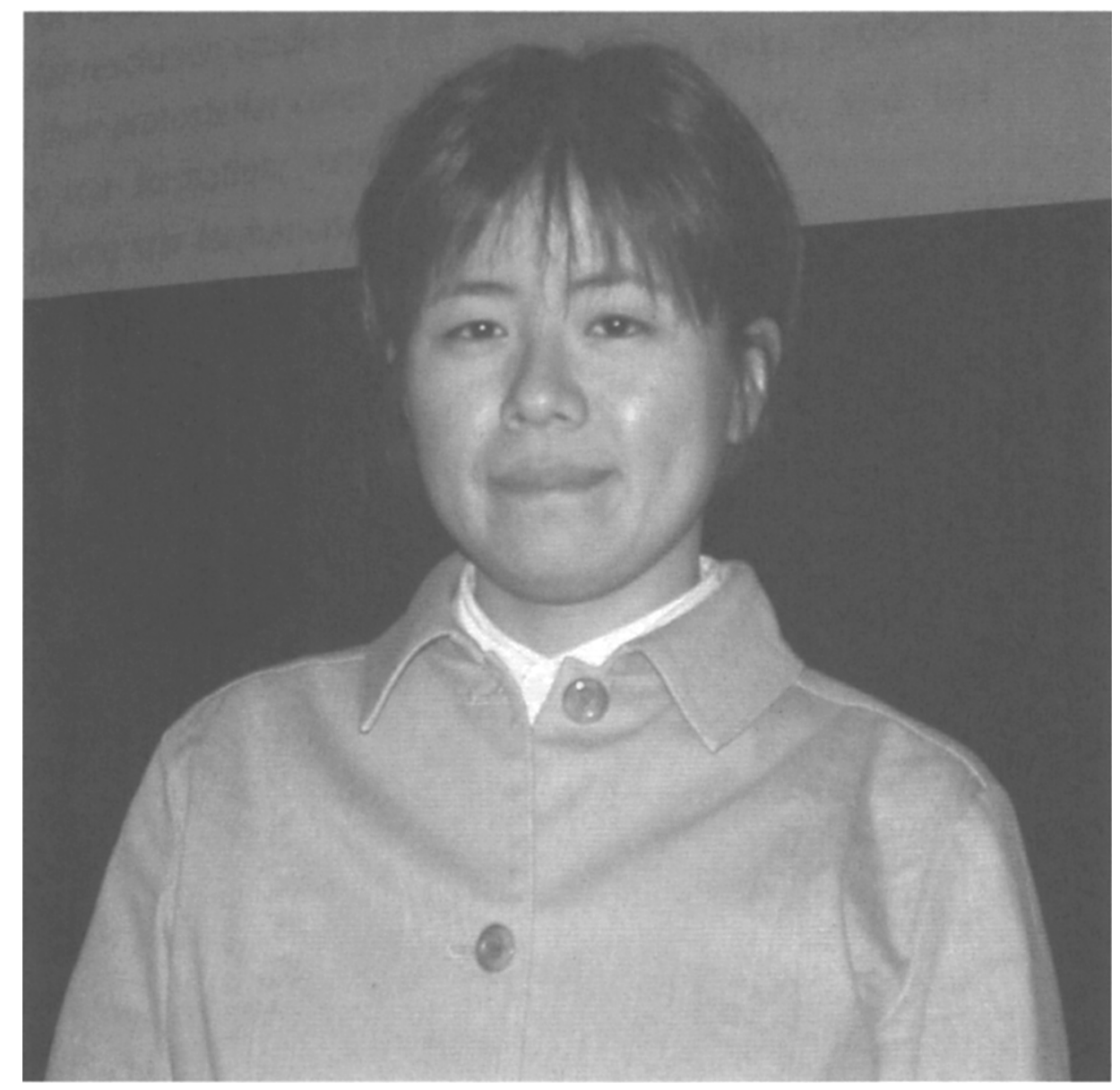

Yohko Tsuboi 